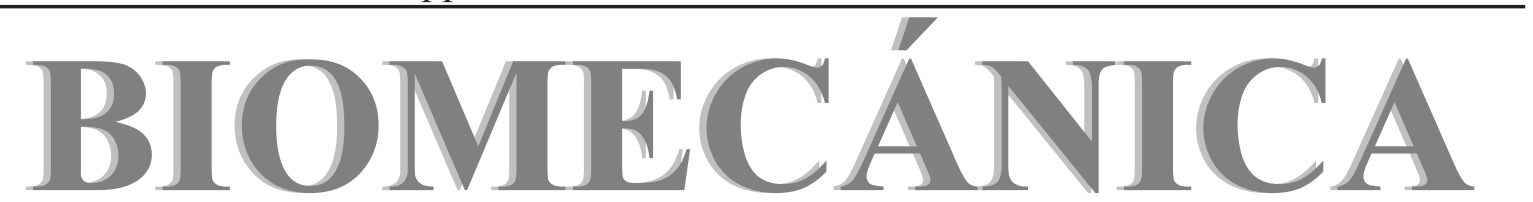

\title{
Tesis doctorales presentadas en España y Portugal en 2014 en el ámbito de la SIBB
}

En esta sección de la revista se listan las Tesis Doctorales presentadas en España y Portugal en el año 2014 y recogidas en la base de datos DART.

DART-Europe es una asociación de bibliotecas de investigación y de consorcios bibliotecarios que trabajan conjuntamente para la mejora del acceso global a las tesis doctorales europeas. DART-Europe facilita a los investigadores un único portal europeo para el acceso a tesis electrónicas (http://www.dart-europe.eu/). DART-Europe está apoyado por LIBER (Ligue des Bibliothèques Européennes de Recherche) y es el Grupo Europeo de Trabajo de la Networked Digital Library of Theses and Dissertations (NDLTD)

17 Área de Biomecánica y Medicina del Deporte

20 Área de Cirugía Ortopédica y Traumatología

25 Área de biomateriales 


\title{
Peso perdido y líquido ingerido y perdido en jugadores de fútbol de categoría infantil, cadete y juvenil en función de la posición ocupada en competición
}

\author{
Autor \\ CASTILLO DÍAZ, ALBERTO \\ Directores \\ GARCÍA JIMÉNEZ, JOSÉ VICENTE; GARCÍA PELLICER, JUAN JOSÉ; \\ YUSTE LUCAS, JUAN LUIS \\ Universidad de Murcia \\ Fecha de lectura: 23 mayo 2014
}

El agua es el protagonista de multitud de funciones vitales en el organismo. Al respecto, y debido a que la práctica del fútbol tan sólo permite la reposición hídrica en determinados momentos, un cierto nivel de deshidratación puede conllevar serios problemas tanto al rendimiento del deportista como a la salud de este. Si a todo esto le sumamos edades pre y puberales, esta situación adquiere un especial interés.

OBJETIVOS El propósito de este estudio ha sido determinar la reposición hídrica y los niveles de deshidratación alcanzados en jóvenes futbolistas en competición en función de la posición ocupada en el terreno de juego.

METODOLOGÍA Es un estudio transversal donde el diseño de la investigación es cuantitativo no experimental de tipo descriptivo, comparativo y correlacional; realizado en 54 jugadores de fútbol de categoría infantil, cadete y juvenil en partidos oficiales de la selección de una comunidad autónoma del territorio español durante el año 2008, seleccionados mediante muestreo de conveniencia, en el que se han analizado variables relacionadas con el estado de hidratación (líquido ingerido, líquido perdido, sudoración, peso perdido, etc.). Las variables de estudio han sido tratadas mediante estadística descriptiva, inferencial (paramétrica y no paramétrica) para llevar a cabo la comparación y, para hallar el tamaño del efecto, se ha recurrido a la d de Cohen y a la regresión logística. Para comprobar la normalidad de las distribuciones se ha comprobado mediante los estadísticos de Kolmogorov-Smirnov y Shapiro-Wilk, estableciendo un valor de $\mathrm{p} \leq 0.05$ para la significación estadística.

RESULTADOS Atendiendo al total de los participantes sin tener en cuenta la posición ocupada, estos presentan un $1 \%$ de peso perdido. Por otra parte, la diferencia de medias entre el líquido perdido $(1104 \pm 554.96$ $\mathrm{ml})$ y el ingerido $(519 \pm 335.93 \mathrm{ml})$ nos indican una pérdida de $585 \mathrm{ml}$. En cuanto a la posición ocupada es la posición de defensa la que presenta valores más elevados de diferencia de medias respecto al líquido ingerido $(549 \pm 348.60 \mathrm{ml})$ y perdido $(1311 \pm 612.45 \mathrm{ml})$ alcanzando una media de $762 \mathrm{ml}$ de pérdida de fluidos y la que mayores niveles de deshidratación alcanza $(1.2 \pm 0.50 \%)$. Respecto a los resultados de la regresión logística, indicar que no hemos hallado relación alguna $(\mathrm{p}>0,05)$ entre las variables de estudio (líquido ingerido, líquido perdido (sudor), porcentaje de líquido repuesto, peso perdido y porcentaje de peso perdido) y el puesto ocupado por los jugadores en el terreno de juego.

CONCLUSIONES A la luz de los resultados expuestos, podemos concluir que los jugadores, en todas las categorías y posiciones analizadas, no reponen las pérdidas de líquidos sufridas durante los partidos en competición provocando un estado de deshidratación progresiva. También destacar que hallamos correlación entre las variables "tiempo total de juego" y "porcentaje de peso perdido" en todas las categorías y en las posiciones de defensa y delantero. Por ello, nuestros resultados invitan a diseñar programas de intervención sobre hábitos correctos de hidratación durante la práctica del fútbol en estas edades atendiendo a la posición ocupada y al tiempo total de juego.

Palabras clave: Deportes, Ciencias del deporte

Enlace de acceso: http://hdl.handle.net/10803/146176 


\title{
Análisis de los métodos más frecuentes de obtención de la victoria en las Artes Marciales Mixtas
}

\author{
Autor \\ GARCÍA BASTIDA, JORGE \\ Director \\ PADULLÉS RIU, JOSEP MARIA \\ Universitat de Barcelona \\ Fecha de lectura: 14 febrero 2014
}

Las Artes Marciales Mixtas (MMA) es uno de los deportes con mayor crecimiento a nivel social y económico en los últimos 20 años. Ya en los antiguos Juegos Olímpicos en Grecia, los deportes de combate disfrutaban de una gran popularidad, pero no fue hasta el año 1993, cuando volvió a aparecer como un deporte de espectáculo. El reglamento de este deporte sufrió diversas variaciones, aumentando el número de reglas asociadas a la competición. Finalmente en el año 2001 se estableció un reglamento regulado por las Comisión Atlética de Nevada, el cual no ha sufrido variaciones desde su creación. La presente Tesis busca analizar los métodos de victoria en MMA, y la relación de dichos métodos con la duración de los combates y con las características físicas de los luchadores. El análisis se ha realizado durante 2 periodos. El primer periodo (grupo 1) correspondió con aquellos combates realizados desde el año 2001 al año 2008, donde no hubo ningún tipo de variación en el reglamento. El segundo periodo (grupo 2) correspondió con aquellos combates realizados durante el periodo comprendido entre los años 1993 y 2001, donde el reglamento sufrió diversas variaciones. Se ha realizado así mismo un análisis comparativo entre ambos periodos. Al comparar ambos periodos encontramos diferencias en la duración promedio de los combates (superior en el grupo 1), en los combates finalizados por decisión de los jueces (superior en el grupo 1) y en los combates finalizados por Submission (superior en el grupo 2). En cuanto a las características físicas de los peleadores, los peleadores pesados obtuvieron un mayor valor porcentual de victorias por $\mathrm{KO}$ o TKO en el primer asalto, mientras que los pesos ligeros e intermedios obtuvieron una mayor frecuencia de victorias mediante Decision en el tercer asalto.

Palabras clave: Deportes de lucha; Artes marciales; Táctica deportiva Enlace de acceso: http://hdl.handle.net/10803/289618 


\title{
El tiempo de reacción específico visual en deportes de combate
}

\author{
Autor \\ ROBLES PÉREZ, JOSÉ JUAN \\ Directores \\ RUIZ BARQUÍN, ROBERTO; MARCOS, RICARDO DE LA \\ Universidad Autónoma de Madrid \\ Fecha de lectura: 15 julio 2014
}

\begin{abstract}
La tesis doctoral que a continuación se presenta estudia el tiempo de reacción específico en artes marciales y cómo se comporta este parámetro en función de los estímulos que se presentan, teniendo en cuenta la especificidad del estímulo y la respuesta. En esta tesis se estudian las diferencias en tiempo de reacción, si se dan y en qué grado entre grupos de expertos con rendimiento deportivo en Karate, Judo y Jiu Jits frente a participantes no practicantes de artes marciales. Los participantes fueron medidos con cámara de alta velocidad mientras respondían a videos con situaciones tácticas determinadas, con distinto grado de transferencia para cada una de las artes marciales, determinando con ello cuales son los factores que intervienen en el sistema perceptivo específico de los expertos de un deporte determinado ante un estímulo, sobre el cual se ha entrenado a lo largo de la carrera deportiva, detectando los preíndices clave del movimiento.
\end{abstract}

Palabras clave: Educación física - Adolescencia - Tesis doctorales; Artes marciales - Tesis doctorales; Deportes Enlace de acceso: http://hdl.handle.net/10486/661009

\section{Epidemiología de las lesiones deportivas en clase de Educación Física}

\author{
Autor \\ GUTIÉRREZ CASTAÑÓN, ELÍAS \\ Directores \\ MARTÍNEZ DE HARO, VICENTE; RAMOS ALVÁREZ, JUAN JOSÉ \\ Universidad Autónoma de Madrid \\ Fecha de lectura: 13 noviembre 2014
}

RESUMEN NO DISPONIBLE

Enlace de acceso: http://hdl.handle.net/10486/662952 


\title{
Bloqueos nerviosos periféricos en la extremidad inferior para la analgesia postoperatoriade la artroplastia total de rodilla
}

\author{
Autor \\ GONZALO PELLICER, IMMACULADA \\ Directores \\ GÓMEZ GÓMEZ, ROBERTO; MARTÍNEZ DELGADO, FERNANDO
}

Universidad de Zaragoza

Fecha de lectura: 4 abril 2014

\begin{abstract}
El control del dolor en el postoperatorio inmediato de la artroplastia total de rodilla (ATR) es básico tanto para la precoz recuperación y movilidad de la extremidad como para el confort del paciente, puesto que se trata de una de las intervenciones más dolorosas que se realizan. Según los últimos estudios publicados, el bloqueo del nervio femoral, ya sea con una punción única o dejando un catéter con bloqueo continuo podría ser la técnica de elección para el control del dolor postoperatorio, aunque no queda del todo claro si los pacientes se beneficiarían además de la adición de un bloqueo del nervio ciático o no.
\end{abstract}

Palabras clave: Ciencias médicas, Cirugía ortopédica, Traumatología

Enlace de acceso: http://zaguan.unizar.es/record/13522 


\title{
Evolución de los ancianos con fractura de fémur: la experiencia en el Vallés oriental
}

\author{
Autor \\ GÓMEZ PALACIO, VICTORIA EUGENIA \\ Directores
GIL ALBAROVA, JORGE; HERRERA RODRÍGUEZ, ANTONIO \\ Universidad de Zaragoza \\ Fecha de lectura: 13 noviembre 2014
}

\begin{abstract}
Las fracturas supracondíleas de húmero en la infancia es un tema de gran interés, presente en libros de traumatología infantil, en las revistas científicas relacionadas, en cursos y congresos. Este interés se centra, por la gran incidencia de esta patología, presente en el día a día del traumatólogo. Con las múltiples controversias que se pueden encontrar. Además es una patología a la que tengo que hacer frente en el día a día de mi actividad profesional en la Sección de Traumatología y Ortopedia Infantil. Las fracturas supracondíleas de humero en la infancia presentan una incidencia del 3-18\%, y se presentan de forma más frecuente entre los 4-8 años de edad, la clínica de los pacientes con esta fractura puede ir desde un leve dolor, hasta gran limitación y deformidad. Es muy importante la correcta exploración clínica del paciente, en búsqueda de lesiones asociadas, como fracturas a otros niveles, que la fractura sea abierta, lesiones neurológicas (trastornos motores y/o sensitivos de los nervios, mediano, radial, cubital y nervio interóseo anterior) y alteraciones vasculares. Para el correcto diagnostico es necesario obtener unas correctas imágenes radiológicas en 2 proyecciones. Con éstas, podemos conocer el tipo de fractura que tenemos que tratar, si es una fractura en extensión o flexión, así como el desplazamiento entre fragmentos. Y con ello podremos clasificar la fractura. Numerosas son las formas de tratamiento de estas fracturas que van desde la colocación de un yeso, el uso de tracción, reducción cerrada y osteosíntesis con agujas de Kirschner en diferentes configuraciones, hasta la reducción abierta. La reducción cerrada y osteosíntesis con agujas de Kirschner es el tratamiento más utilizado para las fracturas desplazadas, pudiendo ser suficiente la inmovilización enyesada en las fracturas no desplazadas. El tratamiento de las fracturas tipo II puede ser más controvertido, a veces se opta por una inmovilización con yeso y en otras la osteosíntesis mediante agujas de Kirschner. Los resultados obtenidos se pueden clasificar con los criterios de Flynn, teniendo en cuenta los resultados estéticos y funcionales. Las complicaciones que se pueden encontrar después del tratamiento de estas fracturas son muy variables: 1) Complicaciones neurológicas, pueden aparecer antes del tratamiento, durante el tratamiento o de forma posterior al tratamiento. Los nervios que se pueden ver afectados son el mediano, radial, cubital y nervio interóseo anterior. 2) Complicaciones vasculares, es fundamental comprobar el pulso y el estado de perfusión de la extremidad, ya que si está alterado, implica la exploración de la arteria braquial, en colaboración con cirugía vascular. 3) Síndrome compartimental, es una de las complicaciones más temidas y devastadoras, que provoca isquemia muscular y nerviosa. Es obligatorio una fasciotomía urgente si se desarrolla. Hay que desconfiar en que no aparezcan las ¿ $\mathrm{P}_{\grave{\iota}}$, ausencia de pulso, dolor, parestesia, palidez, parálisis, poiquilotermia. Porque aunque no estén presentes, se puede desarrollar un síndrome compartimental. 4) Infección, aunque es infrecuente. 5) Necesidad de reintervención, en ocasiones una incorrecta reducción y osteosíntesis, implica que hay que realizar una nueva reducción de la fractura. Ya que la capacidad de remodelación de la zona es muy baja, e implicaría alteraciones en el eje futuras. 6) Deformidad angular, es una complicación tridimensional, y es la más frecuente en las fracturas supracondíleas. Es mejor evitarla con una calidad en el tratamiento. 7) Pérdida de movilidad del codo. 8) Refractura. 9) Miositis osificante. 10) Necrosis avascular de la tróclea. Mención especial merecen las infrecuentes fracturas en flexión, con una incidencia del 2-3\%, por sus características intrínsecas. El tratamiento de este tipo especial de fractura suele ser más difícil, por su complicada reducción que en ocasiones lleva a realizar una reducción abierta. En los últimos 60 años, los cambios introducidos en el tratamiento de estas fracturas, ha supuesto una disminución clara de las complicaciones a nivel vascular, de presencia de deformidades, pero éstas no han desaparecido por completo. Por lo que es interesante el estudio minucioso de esta patología. Aun así hoy en día es una de las patologías más temidas en las urgencias de Hospitales Infantiles. El estudio crítico de series largas de pacientes tratados en centros hospitalarios, con una revisión bibliográfica exhaustiva, permite conocer el estado actual ante esta patología en diferentes centros y hace posible compararla con lo que se realiza en nuestro centro. Con esta comparativa se consigue conocer cuales son los puntos del tratamiento que se están realizando de forma correcta y por lo tanto hay que reforzarlos. Pero a su vez hay que ser conocedores de aquellos puntos mejorables, para el adecuado manejo terapéutico de la fractura, y sus complicaciones, y la consecución de un resultado final satisfactorio para el niño y su familia. Con todo ello se consigue cambiar pautas de actuación mejorables y afianzar las que resultan probadamente eficaces.
\end{abstract}

Palabras clave: Ciencias médicas, Traumatología Enlace de acceso: http://zaguan.unizar.es/record/13300 


\title{
Evaluación del potencial biomédico de nuevos biomateria- les para aplicación en cirugía ortopédica y traumatología
}

\author{
Autor \\ AMAT TRUJILLO, DANIEL \\ Directores \\ MORA HUZMAN, MARÍA JOSÉ; SANTOS RUIZ, LEONOR \\ Universitat de Málaga \\ Fecha de lectura: 1 octubre 2014
}

\begin{abstract}
Las situaciones clínicas relacionadas con fracturas de hueso complejas, tales como fracturas no consolidadas o fracturas patológicas, demandan cada vez más el desarrollo de materiales terapéuticos con la capacidad para inducir la regeneración del tejido óseo, o para reemplazar la pérdida del hueso dañado y restaurar su función. La Ingeniería de Tejidos ha surgido como una alternativa prometedora para reemplazar a las actuales prótesis y sustitutos de hueso. El objetivo de la Ingeniería Tisular, aplicada a la reparación ósea, es producir ex vivo tejido óseo implantable que puede reemplazar al tejido óseo nativo, o estimular su regeneración. Estos implantes consistirían en la combinación adecuada de las células, moléculas osteoinductoras y materiales biocompatibles. Los biomateriales son habituales en Cirugía Ortopédica y Traumatología (COT), ya sea para ayudar a la reparación del hueso o para reemplazarlos y asumir su función biomecánica. La primera aplicación mencionada (ayudar a la reparación), implica el uso de sustitutos de hueso: injertos de materiales bioactivos y reabsorbibles que deben ir desapareciendo a medida que se repara el hueso nativo. Éstos pueden ser de origen natural (hueso del paciente o de donantes); o artificial (cerámicas, polímeros, etc). La segunda aplicación (sustitución) se logra mediante la implantación de prótesis bioinertes, normalmente echas con metales. En cualquier caso, el biomaterial ideal para la reparación ósea debe imitar la matriz extracelular del hueso en cuanto a sus propiedades físicas, mecánicas y químicas. El objetivo del presente trabajo fue evaluar el potencial biomédico, en el contexto de la reparación ósea terapéutica, de diferentes tipos de materiales: las cerámicas silíceas mesoporosas SBA-15 y HA-SBA-15; el biovidrio ICIE-16 y un derivado del mismo (Nitru); y una aleación metálica (Ti6A14V ELI) consolidada en piezas 3D macroporosas mediante fundido por haz de electrones, o tecnología EBM. Los materiales reabsorbibles (cerámicas y biovidrios) se evaluaron in vitro e in vivo para evaluar su biocompatibilidad y sus propiedades osteoconductoras. Las piezas de aleación Ti6A14V ELI se evaluaron in vitro para probar si la superficie resultante del proceso de fabricación por tecnología EBM permiten a las células adherirse, crecer y diferenciarse. Este estudio era necesario porque la osteointegración de prótesis metálicas requiere no sólo una buena integración mecánica, sino también integración tisular, la cual sólo es posible si la respuesta de las células es favorable a la superficie de la prótesis.
\end{abstract}

Palabras clave: Biología Celular, Genética y Fisiología

Enlace de acceso: http://riuma.uma.es/xmlui/handle/10630/8154 


\title{
Inestabilidad de la Artroplastia Total de Cadera
}

\author{
Autor \\ EZQUERRA HERRANDO, LAURA \\ Director \\ ALBAREDA ALBAREDA, JORGE; SERAL GARCÍA, BELÉN \\ Universitat de Zaragoza \\ Fecha de lectura: 23 mayo 2014
}

\begin{abstract}
La artroplastia total de cadera (ATC) es un procedimiento muy frecuente en los Servicios de Cirugía Ortopédica y Traumatología, y aunque su efectividad es muy elevada, no está exenta de complicaciones como son el aflojamiento aséptico, luxaciones, infección y fracturas periprotésicas. A pesar de los avances en las técnicas y modelos protésicos, la luxación sigue siendo la segunda complicación más frecuente después del aflojamiento aséptico en las artroplastias totales de cadera. Se produce en el $0 ; 3 \%$ al $10 \%$ de las artroplastias primarias de cadera y hasta en el $28 \%$ de las artroplastias de revisión. Existen diversas clasificaciones de la luxación de la ATC. Según el tiempo: precoz (3 meses). Según el número: simple o recurrente. Según la dirección: anterior (donde clínicamente se observa el miembro afectado en rotación externa y en extensión) y posterior (miembro inferior en rotación interna y en flexión). Según el mecanismo de lesión: traumático o espontáneo. Y según la etiología, la clasificación de Dorr, importante ya que conociendo la causa de luxación se decidirá la actitud terapéutica (Posicional: no alteración radiológica de componentes sin disbalance de partes blandas (10\%); Malposición de componentes (33\%); Disbalance de tejidos blandos: musculatura, trocánter mayor, vuelo femoral (Offset) (33\%); Combinación de II y III). Por otro lado, se han estudiado ampliamente los factores de riesgo que se consideran asociados a una mayor probabilidad de luxación protésica. Factores relacionados con el paciente como edad, sexo, alteraciones cognitivas o neuromusculares, diagnóstico inicial, cirugía previa. Factores relacionados con la cirugía como vías de abordaje, características de los componentes, mediciones radiológicas pre y postquirúrgicas. Sin embargo, en relación con muchos de ellos sigue existiendo controversia. Dada la alta incidencia de la luxación de la artroplastia total de cadera y la falta de unanimidad en relación con algunos de los factores de riesgo que la favorecen, se plantea este trabajo de investigación clínico-computacional con los siguientes objetivos: clínicos: determinar los factores de riesgo asociados a la luxación protésica y categorizarlos por orden de importancia, y computacionales: simular un modelo protésico con distintos tamaños de componentes y sus rangos de movimiento máximo antes de producirse el choque entre componentes y la luxación, además de correlacionar los hallazgos computacionales con los clínicos para determinar los arcos de movimiento seguros en cada angulación y anteversión del cotilo.
\end{abstract}

Palabras clave: Artroplastia; Prótesis; atc

Enlace de acceso: http://zaguan.unizar.es/record/15514 


\title{
Estudio de la biomecánica del procedimiento de vertebroplastia
}

\author{
Autor \\ GÓMEZ GONZÁLEZ, SERGIO \\ Director \\ FERNÁNDEZ, ENRIQUE \\ Universitat Politècnica de Catalunya \\ Fecha de lectura: 5 noviembre 2014
}

\begin{abstract}
La vertebroplastia (VP) es una técnica quirúrgica que se utiliza para tratar fracturas vertebrales. El procedimiento consiste en inyectar con una cánula un cemento óseo en la vértebra dañada para estabilizar sus propiedades. Los estudios desarrollados se han centrado en: a) buscar soluciones para mejorar el procedimiento de inyección e infiltración en vértebra, mediante simulaciones numéricas y ensayos in vitro con el objetivo de reducir la presión de inyección extravertebral (EV); y b) estudiar cómo afecta la microestructura ósea en la presión intravertebral (IV) y caracterizar la biomecánica del tejido óseo infiltrado. Los estudios de inyectabilidad realizados a partir de estudios analíticos, simulaciones numéricas y ensayos experimentales demuestran que las cánulas diseñadas con perfil cónico requieren menor presión de inyección que las cánulas estándar de sección constante ya que presentan un perfil de velocidades que minimiza el filtrado por presión. Los resultados son importantes porque la presión EV supone más del 95\% de la presión total requerida en el procedimiento de VP. El estudio finaliza con la realización de 4 patentes de dispositivos biomédicos que incluyen una cánula cónica para VP y 3 sistemas de inyección de cemento que aseguran una mezcla homogénea y minimizan el filtrado por presión. Para estudiar el comportamiento del cemento infiltrado se caracterizaron y reconstruyeron en 3D, en primer lugar, diferentes volúmenes de tejido vertebral y de espumas sintéticas empleadas como sustitutos de hueso osteoporótico. Los parámetros histomorfológicos obtenidos demostraron que las espumas evaluadas presentan características distintas en comparación con el hueso sano pero pueden simular correctamente estructuras de hueso osteoporótico. En este sentido, la obtención de modelos $2 \mathrm{D}$ y $3 \mathrm{D}$, a partir de imágenes de tomografía, ha permitido caracterizar mediante dinámica computacional el proceso de infiltración a partir de la evaluación de la permeabilidad y de otros parámetros fluídicos. Los resultados obtenidos mostraron que los parámetros histomorfométricos mantienen elevada correlación con dichos parámetros fluídicos, pudiendo explicar las diferencias estructurales existentes entre las espumas sintéticas y el hueso vertebral. En este sentido, las espumas sintéticas se asemejan a hueso osteoporótico y son incapaces de reproducir la anisotropía característica del hueso trabecular real. En base a los resultados anteriores, se ha desarrollado una metodología de diseño 3D de andamios basada en el método de Voronoi. La metodología reproduce la anisotropía del tejido trabecular real. Los modelos porosos diseñados se han utilizado para evaluar su comportamiento mecánico y de infiltración mediante métodos numéricos. Los resultados confirman la idoneidad de dichos modelos tanto para transporte de masa como de soporte mecánico. De hecho, la gran superficie específica de los nuevos modelos porosos debería facilitar la adhesión y el crecimiento celular. En este sentido, los nuevos modelos podrían ser utilizados como andamios en ingeniería de tejidos. Los nuevos andamios se han diseñado con distinto grado de porosidad modificando el espesor y el ancho trabecular, así como el número de trabéculas. Los resultados computacionales ponen de manifiesto que el módulo elástico y la resistencia de los modelos óseos disminuye de forma más severa cuando la pérdida de "masa ósea" se produce debido a una pérdida en el número de trabéculas activas, en comparación con una situación de adelgazamiento trabecular. Adicionalmente, los estudios demuestran que la forma adoptada por el cemento infiltrado en vértebra influye en la recuperación de su comportamiento mecánico. Las simulaciones indican que una infiltración vertical permite recuperar mejor la resistencia mecánica perdida en comparación con una infiltración horizontal. Todos los resultados obtenidos justifican el empleo de cánulas con aberturas laterales y extremo distal cerrado.
\end{abstract}

Palabras clave: Cirugía; Ensayo de materiales

Enlace de acceso: $\mathrm{http}: / / \mathrm{hdl}$.handle.net/10803/285431 


\title{
Acción de la Vitamina D en la diferenciación osteocondral de hMSCs; potencialidad en la evaluación de biomateriales y vehículos nanoestructurados
}

\author{
Autor \\ SÁNCHEZ VAQUERO, VANESA \\ Directores \\ RUIZ, JOSEFA PREDESTINACIÓN; MANSO SILVÁN, MIGUEL \\ Universitat Autónoma de Madrid \\ Fecha de lectura: 16 enero 2014
}

\begin{abstract}
La osificación endocondral es un proceso de varias etapas conducente a la formación de la mayoría de los huesos del esqueleto. Tiene lugar durante el desarrollo, el crecimiento del individuo, interviene en la remodelación activa del esqueleto, en la reparación después de un insulto y en la regeneración ósea. El hueso no es un tejido estático. A lo largo de la vida se renueva de forma continua, siendo sustituido en su totalidad cada 10 años aproximadamente en el hombre adulto. Con la edad, se produce una osteopenia que conduce a la pérdida de masa ósea y que puede provocar la osteoporosis. Una de las hormonas que juega un papel fundamental, y de la cual se sabe que está descrita su acción en el esqueleto óseo, es la Vitamina D (VD). Los resultados de este trabajo demuestran que la VD reduce la velocidad de proliferación de las células madre humanas mesenquimales, y además, es la responsable de la morfología celular del citoesqueleto. Es capaz de modular la adhesión celular y los genes implicados en la diferenciación osteocondral, la actividad de la fosfatasa alcalina y los factores de transcripción del metabolismo óseo, todo en presencia de prolactina y estrógenos. También se demuestra la participación de la VD en la regulación de la vía Jak-STAT. El tratamiento de los defectos óseos ha sido objeto de controversia durante siglos. El proceso de remodelado es vital para el mantenimiento del sistema esquelético ya que, como resultado de las tensiones y fuerzas a las que está sometido normalmente, acumula microfracturas que han de ser reparadas. Estas áreas dañadas son detectadas y se inicia localmente el proceso de remodelado para su reparación. Para ello se ponen en marcha mecanismos osteoformadores con la finalidad de restaurar el tejido óseo en el lugar de la lesión. Habitualmente, la dinámica del hueso es suficiente para reconstruir los defectos comunes, no obstante, en las pérdidas mayores de masa tisular se hace necesario recurrir al aporte de sustitutivos óseos para obtener la reparación. Por eso, en este trabajo nos hemos centrado en estudiar las células madre humanas mesenquimales y en analizar su comportamiento sobre diferentes soportes de biomateriales. Los resultados sugieren que las células son capaces de diferenciar las múltiples superficies estudiadas como demuestran los cambios morfológicos y de proliferación. Las microesferas de policaprolactona y quitosano permitieron llevar a cabo estudios de condrogénesis, y los materiales micro y nano particulados, como las partículas de silicio poroso infiltradas con cobalto, demostraron no ser citotóxicos para el cultivo celular.
\end{abstract}

Palabras clave: Vitamina D; Huesos; Células madre

Enlace de acceso: https://repositorio.uam.es/handle/10486/660280 


\title{
Evaluación de la Capacidad Osteogénica de las DPPSC (Dental Pulp Pluripotent Stem Cells) sobre diferentes superficies y Biocoating para validar una nueva superfie de Implante Dental
}

\author{
Autor \\ MURTRA SADA, MARÍA CRISTINA \\ Director \\ AL-ATARI ABOU-ASI, MAHER \\ Universitat Internacional de Catalunya \\ Fecha de lectura: 19 marzo 2014
}

\begin{abstract}
Cuando tenemos un implante de Ti convencional, la calidad de su superficie es el pilar fundamental para la osteointegración debido a que sus características topográficas y químicas, son las que controlan la conformación de su capa proteica, y con ello, el sistema de comunicación entre célula-proteína-implante que guiará hacia la deseada osteointegración. Pero en el momento en que a ese implante de Ti convencional se le añade un biocoating, su superficie bioinerte (Ti) se convierte en una superficie bioactiva, donde las biomoléculas que la conforman pasan a ser las activadoras del sistema de comunicación y desencadenantes de la cicatrización, dejando de depender de la adquisición de la capa proteica. Es por ello que nuestro grupo de investigación, con el objetivo de intentar generar un biocoating capaz de asegurarnos la atracción, adhesión, y estimulación necesaria para que se lleve a cabo la osteointegración, desarrollamos un estudio comparativo donde se combinaron 2 tipos de calidades superficiales (desarrolladas por MIS Implants) y 4 biocoatings de composición confidencial (llamados biocoating A, B, C y D), y se concluyó que la utilización de cualquier biocoating fue capaz de aportar una mayor capacidad osteoinductora a la calidad superficial que embebía, y que de todas las combinaciones estudiadas, la que resultó presentar la mayor capacidad osteogénica, y por tanto de mayor capacidad osteointegradora fue el biocoating D sobre la superficie Plates (combinación de mayor calidad superficial y mayor capacidad osteogénica). Además, como la técnica elegida para la obtención de dichos biocoatings había sido a través de una inmersión de 24 horas a 370C (método bioquímico por adsorción), nuestro grupo de investigación creyó necesario probar su capacidad de adhesión y de permanencia antes de iniciar el estudio comparativo. Lo resultados obtenidos probaron que pese a haber utilizando como biomoléculas proteínas propias de la MEC, conocidas por ser poco estables y muy solubles, éstas demostraron tener la suficiente capacidad de adhesión como para permanecer en la superficie de los implantes tras su inserción mecánica a hueso en un 50\% de su cobertura inicial. Y por último, debido a que actualmente los estudios comparativos usados por la industria para validar nuevas superficies o biomateriales aún no está estandarizado, nuestro grupo de investigación decidió utilizar a las DPPSC en este estudio comparativo y además demostrar que poseen una elevada capacidad diferenciadora combinada con una elevada estabilidad genética, un fácil manejo y un fácil acceso (pulpa de terceros molares), que nos permite presentarlas como la línea celular idónea para la protocolización de los futuros estudios de validación de superficies o biomateriales.
\end{abstract}

Palabras clave: Biomateriales; Odontología

Enlace de acceso: http://hdl.handle.net/10803/133080 


\title{
Evaluación de la respuesta celular de células madre adul- tas de origen mesenquimal frente a materiales cerámicos
}

\author{
Autor \\ MÜLLER SÁNCHEZ, CLAUDIA ALEJANDRA \\ Director \\ REINA DEL POZO, MANUEL \\ Universitat de Barcelona \\ Fecha de lectura: 4 junio 2014
}

\begin{abstract}
Las células madre mesenquimales de tejido adiposo (ADSCs) tienen un gran potencial dentro del campo de la ingeniería de tejidos debido a su fácil obtención, capacidad de diferenciación a múltiples linajes, propiedades inmunomoduladoras y producción de factores proangiogénicos y antiapoptóticos. Asimismo, los materiales cerámicos de fosfato de calcio son ampliamente utilizados como biomateriales en la ingeniería de tejidos del hueso, debido a su similitud con la fase mineral del tejido óseo. Además, su combinación con proteínas de matriz extracelular (ECM), factores osteoinductores y otros tipos celulares, puede incrementar la bioactividad del constructo células-biomaterial. A pesar de ello, pocos trabajos existen en la literatura que evalúen la repuesta de células ADSCs frente a biomateriales cerámicos de fosfatos de calcio, empleando recubrimientos con proteínas de ECM y/o el cocultivo de células ADSCs con células endoteliales, como factores claves para el incremento de la inducción de las células hacia linajes osteogénicos. En este sentido, el objetivo del presente trabajo ha sido Evaluar la biocompatibilidad y diferenciación osteogénica de células madre mesenquimales adultas derivadas de tejido adiposo (ADSCs) frente a materiales cerámicos de fosfato de calcio con o sin proteínas de matriz extracelular y células endoteliales. Los resultados mostraron que las células mesenquimales obtenidas de tejido adiposo humano (hADSCs), expresan marcadores característicos de células progenitoras (CD29, CD44, CD73, CD90 Y CD105) y se diferenciaron hacia linajes adipogénico, osteogénico, condrogénico y miogénico. Particularmente las señales de diferenciación osteogénicas de las células hADSCs fueron muy potentes y comparables en gran medida con las de otras líneas de osteoblastos ampliamente utilizadas en el campo de la ingeniería de tejidos tales como las MCT3T3 y hFOB 1.19. Por lo tanto son un excelente modelo celular para la evaluación de biomateriales diseñados con la finalidad de favorecer la regeneración ósea. El diseño de una metodología especial para el cultivo celular sobre biomateriales, permitió la cuantificación eficiente y reproducible del porcentaje de células que se adhieren específicamente al material y el seguimiento de su proliferación. Empleando fibroblastos dérmicos humanos (HDF) se demostró que el biomaterial cerámico KeraOs® $(\mathrm{KO})$ es biocompatible según la ISO 10993-5. El estudio de la respuesta de las células hADSCs frente a diversos biomateriales cerámicos, evidenció que las células se adhieren, proliferan y se diferencian hacia un fenotipo osteoblástico sobre los materiales comerciales Bone Ceramic ${ }^{\circledR}$, Cerasorb $^{\circledR}$ y $\operatorname{KeraOs}{ }^{\circledR}$, aunque no sobre Bio-Oss ${ }^{\circledR}$. Cada material induce una respuesta osteogénica con un perfil particular en la actividad de la enzima fosfatasa alcalina y la expresión de los genes osteonectina y osteocalcina. Aunque los biomateriales solos desencadenan la diferenciación de las células, la adición de factores inductores en el medio de cultivo potencia la respuesta osteogénica. El recubrimiento del material KeraOs® con fibronectina, colágeno o la combinación FN/COL incrementó significativamente la producción de matriz extracelular, la actividad de la enzima fosfatasa alcalina y la expresión de un mayor número de genes asociados a rutas de diferenciación osteogénicas tales como BMP1, BMP2, Runx2, SMAD1, etc. Sin embargo, respecto a las otras dos proteínas, la fibronectina indujo el mayor aumento en la adhesión celular y la respuesta osteogénica. Por otra parte se observó que el cocultivo de células hADSCs con células endoteliales también incrementó el potencial osteogénico de las células hADSCs. Adicionalmente las células endoteliales formaron estructuras tipo capilar y se incrementó la expresión de marcadores angiogénicos tales como VEGF, VE-cad, $\alpha$-SMA y Ang-1. Finalmente se evaluó el efecto del biomaterial KeraOs ${ }^{\circledR}$ combinado con fibronectina y células madre autólogas de tejido adiposo sobre la regeneración ósea de perros Beagles. Similar a lo observado con las mesenquimales humanas (hADSCs), las caninas se adhieren, proliferan y se diferencian hacia un fenotipo osteoblástico, evidenciando su utilidad como modelo para el estudio de la regeneración ósea tanto in vitro como in vivo.
\end{abstract}

Palabras clave: Células madre; Cultivo de tejidos; Ingeniería de tejidos; Materiales biomédicos Enlace de acceso: http://hdl.handle.net/10803/146287 


\title{
Evaluación del potencial biomédico de nuevos biomateria- les para aplicación en cirugía ortopédica y traumatología
}

\author{
Autor \\ AMAT TRUJILLO, DANIEL \\ Directores \\ MORA HUZMAN, MARÍA JOSÉ; SANTOS RUIZ, LEONOR \\ Universidad de Málaga \\ Fecha de lectura: 1 octubre 2014
}

\begin{abstract}
Las situaciones clínicas relacionadas con fracturas de hueso complejas, tales como fracturas no consolidadas o fracturas patológicas, demandan cada vez más el desarrollo de materiales terapéuticos con la capacidad para inducir la regeneración del tejido óseo, o para reemplazar la pérdida del hueso dañado y restaurar su función. La Ingeniería de Tejidos ha surgido como una alternativa prometedora para reemplazar a las actuales prótesis y sustitutos de hueso. El objetivo de la Ingeniería Tisular, aplicada a la reparación ósea, es producir ex vivo tejido óseo implantable que puede reemplazar al tejido óseo nativo, o estimular su regeneración. Estos implantes consistirían en la combinación adecuada de las células, moléculas osteoinductoras y materiales biocompatibles. Los biomateriales son habituales en Cirugía Ortopédica y Traumatología (COT), ya sea para ayudar a la reparación del hueso o para reemplazarlos y asumir su función biomecánica. La primera aplicación mencionada (ayudar a la reparación), implica el uso de sustitutos de hueso: injertos de materiales bioactivos y reabsorbibles que deben ir desapareciendo a medida que se repara el hueso nativo. Éstos pueden ser de origen natural (hueso del paciente o de donantes); o artificial (cerámicas, polímeros, etc). La segunda aplicación (sustitución) se logra mediante la implantación de prótesis bioinertes, normalmente echas con metales. En cualquier caso, el biomaterial ideal para la reparación ósea debe imitar la matriz extracelular del hueso en cuanto a sus propiedades físicas, mecánicas y químicas. El objetivo del presente trabajo fue evaluar el potencial biomédico, en el contexto de la reparación ósea terapéutica, de diferentes tipos de materiales: las cerámicas silíceas mesoporosas SBA-15 y HA-SBA-15; el biovidrio ICIE-16 y un derivado del mismo (Nitru); y una aleación metálica (Ti6Al4V ELI) consolidada en piezas 3D macroporosas mediante fundido por haz de electrones, o tecnología EBM. Los materiales reabsorbibles (cerámicas y biovidrios) se evaluaron in vitro e in vivo para evaluar su biocompatibilidad y sus propiedades osteoconductoras. Las piezas de aleación Ti6A14V ELI se evaluaron in vitro para probar si la superficie resultante del proceso de fabricación por tecnología EBM permiten a las células adherirse, crecer y diferenciarse. Este estudio era necesario porque la osteointegración de prótesis metálicas requiere no sólo una buena integración mecánica, sino también integración tisular, la cual sólo es posible si la respuesta de las células es favorable a la superficie de la prótesis.
\end{abstract}

Palabras clave: Biomaterial, COT, Materiales biomédicos Enlace de acceso: http://hdl.handle.net/10630/8154 


\title{
Methods and techniques for bio-system's materials behaviour analysis
}

\author{
Autor \\ MITU, LEONARD GABRIEL \\ Directores \\ FERRÁNDIZ BOU, SANTIAGO; ROSCA, ILENA CONSTANZA \\ Universitat Politècnica de València \\ Fecha de lectura: 10 febrero 2014
}

\begin{abstract}
In the context of the rapid development of the research in the biosystem structure materials domain, a representative direction shows the analysis of the behavior of these materials. This direction of research requires the use of various means and methods for theoretical and experimental measuring and evaluating. $\mathrm{PhD}$ thesis "Methods and means for analyzing the behavior of biosystems structure materials" shall be given precedence in this area of research, aimed at studying the behavior of polymeric materials and composites biosystems structure and in particular the skeletal structure biosystem. Therefore, it is developed a specific method of research based on the development of theoretical models for the prediction of the mechanical, thermal and machinability properties of these materials. There are used Moldflow, Solidworks and Ansys software types. In order to validate the theoretical research were designed and conducted experimental research on the mechanical properties and the behavior of the polymeric biomaterials represented by ABS, UHMWPE, HDPE, PA, PC, PET, PP, PP_GF$30 \%$ and composite materials with polymeric thermoplastic matrixes from the skeletal biosystem $;$ s structure. In order to analyze the theoretical and experimental correlations, the experimental data were processed using the statistical analysis software programs SPSS v17, v8 Origin, Palisade Decision Tools. In conclusion, the thesis represents a technic, scientific and efficient support for analyzing the behavior of the new polymeric and composite materials from the biosystem structure.
\end{abstract}

Palabras clave: Biosystems; Composites; Prosthetic blades

Enlace de acceso: http://riunet.upv.es/handle/10251/35445 


\title{
New approaches in calcium phosphate cements and ceramics for bone regeneration
}

\author{
Autor \\ GALLINETTI, SARA \\ Directores \\ CANAL BARNILS, CRISTINA; GINEBRA MOLINS, MARIA PAU \\ Universitat Politècnica de Catalunya \\ Fecha de lectura: 19 septiembre 2014
}

\begin{abstract}
El hueso es uno de los tejidos más trasplantados del cuerpo. Sólo en Europa, se cuentan alrededor de un millón de cirugías de reconstrucción ósea anualmente. La estimación del mercado global de los sustitutos óseos es aproximadamente de cinco billones de Euros por año, con un 10\% de crecimiento anual debido al envejecimiento de la población. Debido a los problemas asociados a los injertos biológicos, la investigación y el desarrollo de materiales sintéticos y biocompatibles (Biomateriales) ha experimentado un gran auge. Aunque la mayoría de sustitutos sintéticos disponibles poseen algunas de las características de los autoinjertos, hasta el momento ninguno reúne todos los beneficios del hueso del propio individuo. Dentro de los biomateriales para regeneración ósea, los fosfatos de calcio han sido de gran interés debido a su composición química similar a la del hueso. Sin embargo, aún se requieren mejoras en distintos aspectos de estos materiales. El objetivo principal de esta Tesis Doctoral es contribuir a la mejora de las propiedades de los fosfatos de calcio para la regeneración ósea, con un interés especial en los cementos de fosfato de calcio. La Tesis investiga diferentes estrategias para el desarrollo de materiales para la sustitución ósea, novedosos y con propiedades mejoradas respecto a los actuales. La Tesis comprende tres partes principales: i) Cementos bifásicos de fosfato de calcio (BCPCs), constituidos por materiales con diferente solubilidad; ii) Fosfatos de calcio reforzados con fibras (FRCPCs), para la mejora de las propiedades mecánicas; iii) Andamios macroporosos para la liberación de una sal de simvastatina. En la primera parte de la Tesis, se describe el desarrollo de BCPCs compuestos por hidroxiapatita deficiente en calcio (CDHA) y fosfato tricálcico ß (ß-TCP). Estos materiales derivan de la reacción de las mezclas de dos polimorfos de fosfato tricalcico (TCP) con diferente solubilidad (a-TCP y $\beta-T C P)$ y, en esta tesis, se caracterizan su fraguado, sus propiedades mecánicas y degradación. En la segunda parte, se han desarrollado nuevos FRCPCs con especial atención hacia la mejora de la adhesión entre fibras y matriz, con el objetivo de mejorar la transferencia de carga entre ellos y por tanto, las propiedades mecánicas del compuesto. Se han investigado distintas estrategias. La primera de ellas basada en la investigación de materiales con una fase común (o con alta afinidad química) entre las fibras y la fase liquida del cemento; de esta manera se pretende crear un enlace más fuerte entre las fibras y la matriz. En un primer material se incorporó un $1 \mathrm{w} / \mathrm{v} \%$ de Trimetilo de quitosán (TMC) en la fase líquida del cemento que a su vez se reforzó con fibras de quitosán. En un segundo grupo de materiales, se añadió un 10 v/v\% de ácido láctico (LA) a la matriz del cemento junto con hilos discontinuos de ácido poliláctico (PLLA). Estos cementos también se caracterizaron biológicamente por medio de células osteoblásticas MG63. La segunda estrategia investigada en los FRCPCs se basa en la modificación superficial de las fibras de PLLA con plasma de baja temperatura con el fin de mejorar sus propiedades de mojado. Las fibras se trataron con plasma de oxigeno de baja presión a distintos tiempos y se incorporaron a la matriz de cemento, y se caracterizaron tanto las modificaciones superficiales de las fibras como las propiedades del cemento. La tercera parte ha consistido en el desarrollo de andamios macroporosos obtenidos a baja (CDHA) o alta (ß-TCP) temperatura para ser utilizados como formas de liberación de una sal de simvastatina (SVA), con propiedades osteogénicas y angiogénicas. Para conseguir modular la liberación del fármaco se recubrieron los andamios cargados con SVA con un copolímero de PCL:PEG mediante polimerización por plasma. Se caracterizaron las propiedades tanto del material como del recubrimiento y se evaluó la liberación del fármaco.
\end{abstract}

Palabras clave: Biomaterials; fosfatos de calcio

Enlace de acceso: http://hdl.handle.net/10803/279558 